\title{
Lipidomics and Free Radical Modifications of Lipids
}

\author{
Chryssostomos Chatgilialoglua ${ }^{\mathrm{a}}$, Carla Ferreriª, Albin Hermetter ${ }^{\mathrm{b}}$, Emmanuel Lacote ${ }^{\mathrm{c}}$, Branka \\ Mihaljevićd $^{d}$, Athanassios Nicolaides ${ }^{\mathrm{e}}$, and Athanassia Siafaka-Kapadai ${ }^{\dagger}$
}

\begin{abstract}
The free-radical-induced modification of biologically relevant molecules is an active field of interdisciplinary research, spanning from chemistry to biochemistry, biology and medicine. Lipid modifications are also attracting attention for their relationship with structural and functional roles in physiological and pathological conditions of living organisms. The discipline of lipidomics studies the lipid behavior in an innovative and multidisciplinary context. Combining lipidomics with free radical chemistry, the research field becomes an ideal setting for the chemical biology approach, to study the basis of molecular mechanisms and chemical reactivity and connect them with free-radical-based processes occurring in the biological environment. This paper will give an overview of the approaches for studying free radical processes on lipids and some biological consequences, which represent also subjects of interdisciplinary collaborations among European research groups and contribute to the general topic of the COST Action 'Free Radicals in Chemical Biology'.
\end{abstract}

Keywords: Lipid isomerization · Lipid peroxidation · Lipidomics · Peroxy lipids · Trans fatty acids

\section{Introduction}

Lipids are molecules with different structures having in common the feature of water insolubility. These molecules have very important biological roles, with structural, functional and signaling activities that have nowadays regained the attention of life science research. The novelty brought about by the approach of lipidomics in the last

${ }^{*}$ Correspondence: Dr. C. Ferreri ${ }^{\mathrm{a}}$

Tel.: +390516398289

Fax: +390516398349

E-mail: cferreri@isof.cnr.it

aISOF, Consiglio Nazionale delle Ricerche

Via Piero Gobetti 101

40129 Bologna, Italy

bInstitute of Biochemistry

Graz University of Technology

Petersgasse 12/II, A-8010 Graz, Austria

'Université Pierre et Marie Curie

Institut de chimie moléculaire (FR 2769)

Laboratoire de chimie organique (UMR CNRS 7611)

4 place Jussieu, C. 229, 75005 Paris, France

dDivision of Materials Chemistry

Radiation Chemistry and Dosimetry Laboratory

Rucker Bošković Institute

Bijenička 54, 10000 Zagreb, Croatia

eDepartment of Chemistry University of Cyprus

POB 20537

Nicosia 1678, Cyprus

fDepartment of Chemistry

University of Athens

Panepistimiopolis, 15771 Athens, Greece years consisted of a dynamic vision of lipids existing in living organisms. ${ }^{[1,2]}$ In fact, lipidomics considers not only the structural and functional roles played by lipids, but also their in vivo transformations and modifications by metabolic or degradation pathways, as well as their biological consequences. In this context, in the last decades the effects of free radical processes were recognized as being important in physiological and pathological conditions. The first radical process on lipids to be recognized was peroxidation, a transformation mainly occurring at the expense of fatty acids containing bisallylic positions, i.e. more than one double bond. This process can occur to natural substrates such as triglycerides, phospholipids, eicosanoids. The study of lipid peroxidation was first approached in chemistry, ${ }^{[3]}$ then has involved, and still it does, several disciplines of life sciences.[4] This topic can be certainly considered as a successful example of the fruitful relationship between chemical and biological investigations. More recently, the radicalbased process of lipid isomerization has been evidenced that occurs to unsaturated lipids and consists of the transformation of the naturally occurring cis geometry of lipid double bonds to the corresponding geometrical trans isomer.[5,6] Also this process involves several lipid structures and takes advantages from being studied in a multidisciplinary research context, in order to use chemical knowledge as the molecular basis for biological and medical investigations.

Lipidomics of free-radical-based modifications can furnish the ideal context for a chemical biology approach, fostering collaborations among different disciplines and expertise. Free radical behavior in living systems can be fully understood only by combining chemical with biological properties and conditions, to improve the comprehension of molecular mechanisms for the interpretation of biological results.

Depending on the role played by the lipid structures, membrane lipidomics and mediator lipidomics can be distinguished. In the first case, the lipid role is targeted for its contribution to membrane composition, correlating structural variations and change of biophysical properties with the effects on the membrane assembly and functioning. In the mediator lipidomics, the focus is on signaling pathways derived from lipids and the change of molecular interactions that can have influence on a biochemical response.

As far as the methodologies of lipidomic studies are concerned, biomimetic models can be useful to explore lipid reaction outcomes and products in a simplified environment. These models can provide basic molecular mechanisms and libraries that can be transferred for the evaluation of more complex biological systems, from cell cultures to living organisms. 


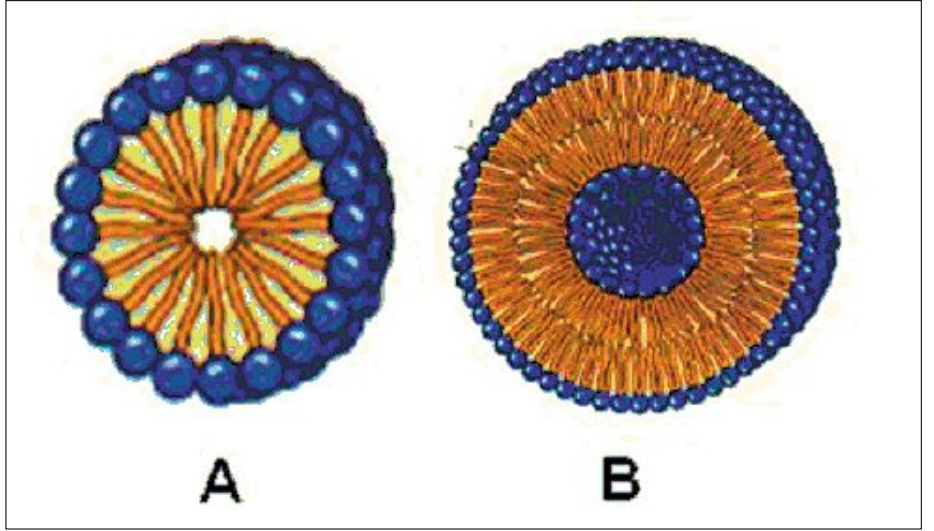

Fig. 1. Examples of lipid aggregation: micelle (A) and liposome (B)

The study of fatty acid reactivity in model systems can be performed under different experimental conditions, such as:

- homogeneous solutions, taking into account that lipids are hydrophobic, therefore soluble in organic solvents such as alcohols, chloroform, hexane, etc.

- heterogeneous systems, using an aqueous environment, where lipid molecules organize themselves spontaneously. The two main organization modes are micelles and liposomes. Free fatty acids organize themselves in micelles, whereas phospholipids form liposomes (Fig. 1). This latter system is characterized by the presence of an aqueous cavity and is closer to a biological model for lipid aggregation. In fact, liposome vesicles are widely accepted as model for the lipid bilayer of cell membranes.

In this paper radical processes of lipid peroxidation and isomerization and some biological consequences will be described, highlighting the work done by the authors and the context of future collaborations in the frame of the COST Action 'Free Radicals in Chemical Biology'.

\section{Lipid Peroxidation}

Polyunsaturated fatty acids (PUFA) are constitutive parts of lipids, present in all biological membranes and lipoproteins. In PUFA the most sensitive site to oxidative attack is the bisallylic position between two double bonds. Detailed studies of the products and mechanism of peroxidation were initiated in the 70s by several research groups. ${ }^{[7-9]}$ The first products to be identified were the hydroperoxides derived from the corresponding peroxyl radicals (Scheme 1).

The mechanism of lipid peroxidation (a radical chain reaction) starts with the abstraction of hydrogen atom producing the bisallylic (or pentadienyl) radical $L^{\bullet}$. The propagation steps are illustrated in reactions 1 and 2 . The reaction of $\mathrm{L}^{\bullet}$ with oxygen is close to a diffusion-controlled process, but is also reversible. Indeed, the peroxyl radical can undergo a very rapid fragmentation $\left(10^{6} \mathrm{~s}^{-1}\right)$, that can also serve as a useful clock for $\mathrm{H}$-atom transfer from antioxidant molecules. Peroxyl radicals $\mathrm{LOO}^{\bullet} \mathrm{can}$ abstract a hydrogen atom to produce lipid hydroperoxide $(\mathrm{LOOH})$ together with 'fresh' $\mathrm{L}^{\bullet}$ radicals to continue the chain (reaction 2). Termination steps occur either by radical-radical combination or by attacking other molecules, such as an antioxidant ( $\alpha$-tocopherol) or proteins.

$$
\begin{aligned}
& \mathrm{L}^{\bullet}+\mathrm{O}_{2} \rightleftharpoons \mathrm{LOO}^{\bullet} \\
& \mathrm{LOO}^{\bullet}+\mathrm{LH} \stackrel{k_{p}}{\longrightarrow} \mathrm{LOOH}+\mathrm{L}^{\bullet}
\end{aligned}
$$

The distribution of hydroperoxides formed depends on the efficiency and concentration of the hydrogen atom donor present. For example, when oxidation of linoleic acid occurs in the presence of $\alpha$-tocopherol ( $\alpha$-Toc, $<10 \mathrm{mM})$, a trans,cis mixture of conjugated hydroperoxides is the major product.[10]

The ability of PUFA to undergo chain oxidation (autoxidation) is termed oxidizability, $k_{\mathrm{p}} / \sqrt{2 k_{t}}$ (where $k_{\mathrm{t}}$ are the rates of radical-radical termination reactions), a composite quantity defined by the ratio of the propagation rate constant and the square root of the termination rate constant.

The classical rate law for autoxidation in homogeneous solutions has been shown to extend to micelles and lipid bilayers. Linoleic acid, taken as typical example of unsaturated fatty acid, has a similar oxidizability in different media as determined by different procedures (0.02-0.04 $\left.\mathrm{M}^{-1 / 2} \mathrm{~s}^{-1 / 2}\right)$. ${ }^{\text {[9] }}$

The systematic determination of oxidizability in the extended homologous series of
PUFA indicate an increase with increased number of bisallylic carbons. The relationship in the series linoleic acid/linolenic acids/arachidonic acid/docosapentaenoic acid/docohexaenoic acid has been shown to be $1: \sqrt{ } 2: 2: 2 \sqrt{ } 2: 4 .{ }^{[11]}$ Contrary to previous measurements, which were based on continuous monitoring of the consumption of oxygen, in this work the radiation-induced peroxidation of PUFA in bulk was followed by direct measurement of $\mathrm{LOOH}$ formed.

The products of lipid degradation and decomposition are commonly expressed as conjugated dienes, ${ }^{[12]}$ as well as aldehyde end-products, which are used to assess the occurrence of oxidative stress. ${ }^{[13]}$ Spectrophotometric quantification of $\mathrm{LOOH}$ is used to follow accurately the initial stages of the process. ${ }^{[14]}$

\subsection{Metal-mediated Lipid Peroxidation}

Extensive studies have been performed on metal-mediated lipid peroxidation, such as copper or iron-induced peroxidation, since it has a relationship with membranes and human diseases. ${ }^{[15-17]}$ However, the mechanism by which iron, as well as other metals, initiates lipid peroxidation is still under debate. In the iron case, one of the theories proposes induction of lipid oxidation by hydroxyl radicals $\left({ }^{\bullet} \mathrm{OH}\right)$, formed via autoxidation of $\mathrm{Fe}$ (II) and formation of hydrogen peroxide (reaction 3 ), ${ }^{[18]}$ and the subsequent ferrous-catalyzed $\mathrm{H}_{2} \mathrm{O}_{2}$ cleavage to ${ }^{\circ} \mathrm{OH}$ by the Fenton reaction (reaction 4$)$.

$$
\begin{aligned}
& \mathrm{Fe}^{2+}+\mathrm{O}_{2}^{\bullet-}+2 \mathrm{H}^{+} \rightarrow \mathrm{Fe}^{3+}+\mathrm{H}_{2} \mathrm{O}_{2} \\
& k=2 \times 10^{8} \mathrm{M}^{-1} \mathrm{~s}^{-1} \\
& \mathrm{H}_{2} \mathrm{O}_{2}+\mathrm{Fe}^{2+} \rightarrow \bullet \mathrm{OH}+\mathrm{OH}^{-}+\mathrm{Fe}^{3+}
\end{aligned}
$$

Hydroxyl radicals formed subsequently react with polyunsaturated lipids leading to the formation of LOOH via the propagation steps of lipid oxidation process (reactions 1 and 2). The data accumulated over the past few decades have shown that hydroxyl radicals can be dismissed as the first initiator of lipid peroxidation.[19] Minotti and Aust reported the necessity of the couple $\mathrm{Fe}(\mathrm{II}) /$ $\mathrm{Fe}(\mathrm{III})$ for the lipid oxidation process to take place, as neither Fe(II) nor Fe(III) alone could promote the oxidation of polyunsaturated lipids.[20] To explain the requirement of the $\mathrm{Fe}(\mathrm{II}) / \mathrm{Fe}$ (III) couple as initiator of the lipid oxidation process, mixed-valence iron-oxygen and perferyl-oxo complexes

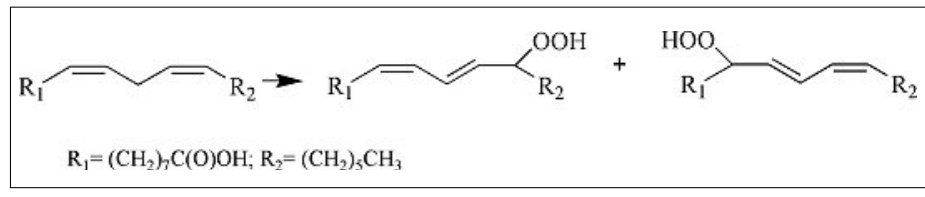

Scheme 1. Lipid peroxidation of linoleic acid with formation of hydroperoxides 
were proposed to be formed. However, until now there is no conclusive evidence for the hypervalent iron complex as a proposed initiator.

Numerous studies were carried out in biological or model systems concerning autoxidation induced by $\mathrm{Fe}$ (II) as mechanism of the reactive oxygen species formation, simultaneously neglecting the role of iron and lipid hydroperoxides preformed in these systems by specific mechanisms. Since the reactions of $\mathrm{Fe}(\mathrm{II})$ with $\mathrm{LOOH}$ are much faster than those of $\mathrm{Fe}(\mathrm{III})$ with LOOH, hydroperoxides undergo breakdown by $\mathrm{Fe}$ (II) leading to the formation of alkoxyl and alkyl radicals (reactions 5 and 6 , respectively), followed by propagation of the lipid oxidation process through reactions 1 and 2.

$$
\mathrm{Fe}^{2+}+\mathrm{LOOH} \rightarrow \mathrm{LO}^{\bullet}+\mathrm{OH}^{-}+\mathrm{Fe}^{3+}
$$

$\mathrm{LO}^{\bullet}+\mathrm{LH} \rightarrow \mathrm{LOH}+\mathrm{L}^{\bullet}$

The pre-existing $\mathrm{LOOH}$ has been shown to occur widely in biological samples or model membranes and to play an essential role in iron-mediated lipid peroxidation. The LOOH-dependent initiation, which is also referred to as 'secondary initiation' initially develops two kinds of free radicals, lipid alkoxyl radicals $\left(\mathrm{LO}^{\bullet}\right)$ and lipid peroxyl radicals $\left(\mathrm{LOO}^{\bullet}\right)$. Alkoxyl radicals are highly reactive. It has been reported that $\mathrm{Fe}$ (II) can scavenge alkoxyl radicals at a rate constant of $3.4 \times 10^{8} \mathrm{M}^{-1} \mathrm{~s}^{-1}$.[21] This reaction is fast compared to reaction $5(\sim$ $\left.1.3 \times 10^{3} \mathrm{M}^{-1} \mathrm{~s}^{-1}\right)^{[22]}$ and can suppress the initial formation of $\mathrm{LO}^{\bullet}$ or even terminate the chain reaction of lipid peroxidation in excess of $\mathrm{Fe}$ (II) concentration.

\subsection{Oxidative Modification of Lipids: Biological Significance}

The first demonstration of free radical oxidation of membrane phospholipids was given in 1980,[23] leading to a new fruitful era with a continuous flow of work devoted to chemistry, biochemistry and medicine. ${ }^{[24]}$ As already mentioned, the simulation of such transformations can be approached by using models of fatty acid aggregates in aqueous media, such as micelles and liposomes (Fig. 1), as well as complexes of lipids with proteins. Linoleic acid has been frequently used as a model fatty acid in the studies of lipid peroxidation, but also arachidonic acid is used, since both are the most abundant PUFA found in humans.

Characteristic oxidative stability of PUFA in organized systems may be correlated with the specific physical properties of PUFA. Their tight conformation may result in the selective attack of free radicals and/or oxygen at the bisallylic position in PUFA. Consequently, it would be expected that there are some differences in the distribution of positional isomers of lipid hydroperoxides between the oxidation in aqueous micellar solutions of PUFA and autoxidation of neat substrate in the air.

Since the first chemical studies, it has been demonstrated that in the autoxidation of neat PUFA the main products were isomers of the lipid hydroperoxides (see Scheme 1). The stereoisomeric alcohols, 9-hydroxy10,12-octadecadienoic acids (9-HODEs) and 13-hydroxy-9,11-octadecadienoic acids (13-HODEs), have been found in vivo as enzymatic products of linoleic oxidation, in variable amounts depending on the tissues. ${ }^{[25]}$ Fatty acids with more than two double bonds are able to give also other products, such as cyclic endoperoxides which can also be products of in vivo enzymatic transformation. The effects of peroxidized fatty acids have been checked by administering such compounds to animals. [26,27] It is not surprising that the toxicity can be considered overall low, because lipid oxidation is part of natural biochemical pathways. Lipoxygenases, cyclooxygenases and cytochrome P450 enzymes are involved in such transformations and knowledge on their interactions with substrates is increasing. ${ }^{28,29}$ The oxidized products are well known as bioactive compounds that influence metabolism and enzymatic systems, and below a few examples will be given. It must be added that there are excellent scavengers of peroxyl radical species that act in vivo to balance the physiological production, and only when the cells are severely wounded, the peroxide radical production cannot be controlled, thus becoming harmful. This is the reason that oxidative pathways became a very active field of interdisciplinary research, because of their correlation with disease.

Perhaps, the most well-known process is the oxidation of LDL (low density lipoprotein) that is recognized as an important contributor to the pathogenesis of atherosclerosis. ${ }^{[30,31]}$ The phospholipid part of LDL, that accounts for up to $20-25 \%$ of the whole particle (by weight), is the primary target of oxidation in an LDL particle. In vitro oxidation of LDL has led to the isolation of several oxidized phospholipids, and with the development of HPLC methods coupled with ion-spray mass spectrometry, identification of these primary products in the early stages of LDL oxidation has been performed. ${ }^{[32]}$ Biological assays showed that they are bioactive compounds, involved indeed in the biological events associated with atherosclerosis. In fact, the uptake of oxidized low-density lipoprotein by the cells of the vascular wall plays a key role in atherogenesis. The modified particles either inhibit or induce (programmed) cell death, depending on the extent of lipoprotein oxidation and dose. These effects can be initiated by minimally modified LDL (mmLDL) in which only the lipids are oxidized. The contribution of the individual lipids to the toxic effect of mmLDL on vascular cells is far from clear. Current interests focus on the cytotoxic effects of the phosphatidylcholines 1-palmitoyl-2-(5-oxovaleroyl)-sn-glycero3-phosphocholine (POVPC), 1-palmitoyl2-glutaroyl-sn-glycero-3-phosphocholine (PGPC) and their 1-O-alk(en)ylether analogs that are oxidation products of choline glycerophospholipids containing arachidonic acid in position $s n-2$ of glycerol. The research aim is to identify the role of these compounds in cell death as a consequence of apoptosis and/or necrosis. The 1-O-alk(en)yl (ether) analogs of PGPC and POVPC are studied since ether phospholipids also represent important lipoprotein and membrane components. To understand the molecular mechanisms of lipid-induced cell death, the uptake and stability of the oxidized phospholipids in cells has to be determined, in order to identify their primary targets, measure their apoptotic and necrotic effects, and analyze the intracellular signaling components. ${ }^{[33]}$

An original approach that is currently under examination is to combine the properties of membranes with those of inorganic polyanions, such as synthetic polyoxometallates (POM) which are nanoclusters of highly oxidized early transition metals and oxygen with important biological activities. ${ }^{[34]}$ The origin of such activities are still under exploration, but given the anionic character of those compounds, it appears that membrane crossing is an issue that needs to be addressed for deeper understanding of the systems. Upon reduction at the cell membranes POM might also generate radicals that would produce effects to the cells. POM structures can contain an 'arm' to be used as a linker (Fig. 2), and conditions have been developed to attach various molecules to the acid moiety by coupling reactions.[35-37]

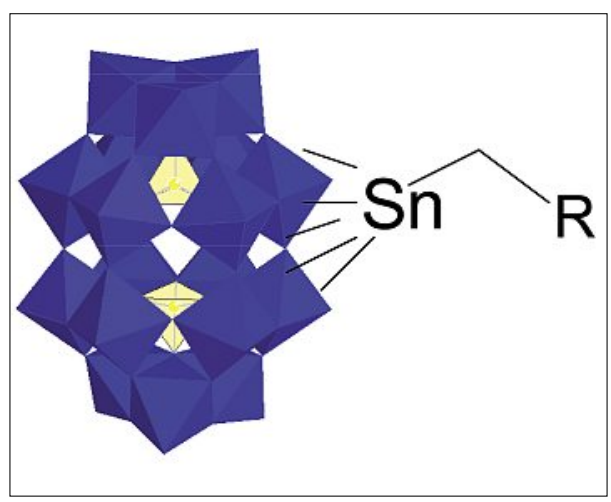

Fig. 2. Polyoxometalate structures to be functionalized

The synthesis of POM-containing fluorescent labels can be then envisaged for the study of interaction with biological structures such as membranes, or inducing 
cell responses, thus affecting cell growth and death.

The scenario regarding modifications of unsaturated lipids would not be complete without mentioning the oxidation products of cholesterol esters of linoleic and arachidonic acids. These esters are incorporated into VLDL that are then transformed into LDL. When this LDL is transferred to endothelial cells, toxic products are liberated and induce cell damage. The damage is combined with structural changes that influence neighboring cells and cause an influx of $\mathrm{Ca}^{+2}$ ions, with activation of phospholipase first, that is responsible of the free fatty acid liberation from membranes, and secondly to lipoxygenase enzymes that produce lipid peroxides. When the level of free PUFA obtained from the phospholipase activity exceeds certain limits, lipoxygenase commits suicide, liberating iron ions. These ions react with lipid peroxides, a production of peroxyl radicals via non-enzymatic reactions, which are able to attack any biological compounds, inducing severe damage.[38]

The formation of lipid peroxides, similar to that occurring in cardiovascular diseases, can be observed in inflammatory and neurodegenerative diseases, indicating a close relationship between the inducers of all these events. The formation of oxidized lipids in membranes seems to influence the channels across the bilayer, and causes an influx of $\mathrm{Ca}^{+2}$ ions, so that phospolipase enzymes are activated. The cleavage of phospholipids occurs and it will cause damage as described above.[39]

What are the most sensitive membrane phospholipids to oxidative damage has been the subject of several investigations, using model liposomes as well as living organisms. In rats, ischemia-reperfusion injury to cardiac myocytes involved membrane damage with the most pronounced effects on cardiolipin (Fig. 3), a lipid which is mostly present in mitochondria, with its oxidation products being involved in apoptosis and signaling. ${ }^{[40,41]}$ One of the most indicative experimental protocols for investigating the damage of the membrane lipids uses irradiation. Employing the oxidative lipidomics to qualitatively and quantitatively characterize phospholipids peroxidation in the small intestine of mice exposed to total body irradiation (TBI) of 10 and $15 \mathrm{~Gy}$, in a recent work has been established that two anionic phospholipids - cardiolipid in mitochondria and phosphatidylserine outside mitochondria - are the most involved in oxidation. Phosphatidylcholine and phosphatidylethanolamine did not reveal any oxidative stress responses despite the presence of highly oxidizable docosahexaenoic fatty acid residues in their structures. ${ }^{[42]}$ Therefore, the sensi-

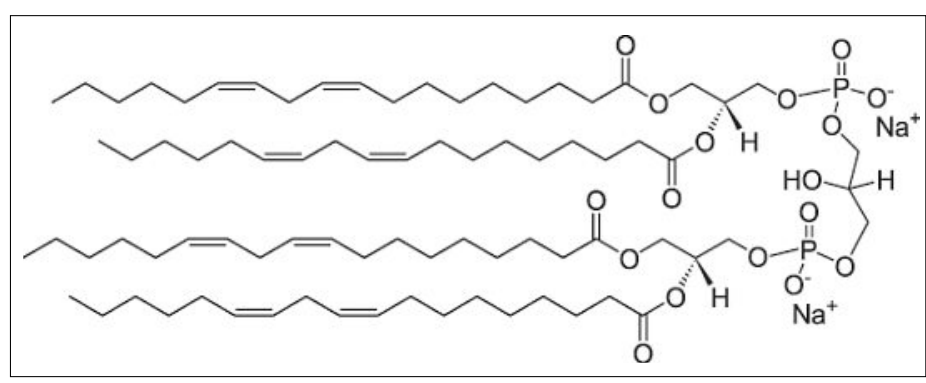

Fig. 3. Cardiolipin structure

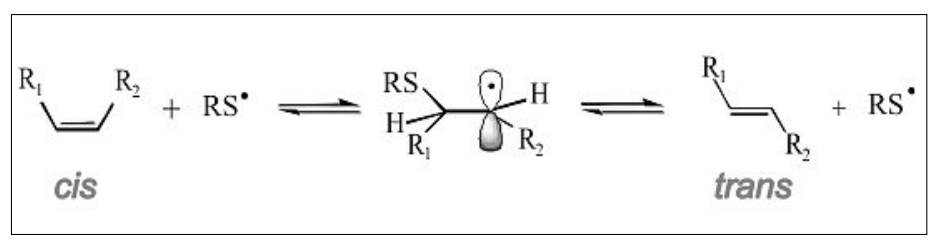

Scheme 2. Cis-trans isomerization by thiyl radicals

tivity of the different lipid molecules can vary depending on the biological context and location.

It is however clear that lipid oxidation in vivo has a very important target in membrane phospholipids components. In particular, being the polyunsaturated fatty acid usually located in the $s n-2$ position of the glycerol backbone, this is the position that is modified in oxidized phospholipids. ${ }^{[43]}$ It is important to recall at this point that the above described lipid peroxides are the starting products of this damage. In fact, from the intermediate peroxyl radicals, a cascade of radical reactions can occur, including hydrogen abstractions and fragmentations, generating truncated phospholipids and different types of low molecular weight aldehydes. The aldehydes, such as malondialdehyde (MDA), 4-hydroxynonenale (HNE) and other related unsaturated aldehydes, have been found as products of the cleavage of hydroperoxides. MDA was the focus of attention in lipid peroxidation for many years, which motivated the use of the thiobarbituric acid (TBA) to assay the peroxidation in vitro. ${ }^{[44]}$ Also HNE was investigated for its role in this degradation process, ${ }^{[45]}$ and found to be particularly toxic to mitochondria.[46] These structures containing a double bond, an aldehyde function and a hydroxyl group are chemically very reactive, and they can easily form adducts with many functional groups, such as thiol and amino groups, widely present in the biological environment, for example in proteins and the nucleotidic components of DNA. Therefore, the adducts have a mutagenic potential, and in general very strong toxicity effects. ${ }^{[47,48]}$ These compounds can induce the production of antibodies, and this has led to the increased use of HNE or MDA antibodies that are able to visualize their adducts present in a tissue. This is very useful to demonstrate the importance of these reactions in diseases and ageing. As examples, modification of proteins with the lipid peroxidation product 4-HNE has been detected in human peripheral blood lymphocytes, using two- dimensional gel electrophoresis followed by Western blotting with an antibody recognizing 4-HNE protein adducts. ${ }^{[49]}$ Analogously, such biomarkers have been used to detect the occurrence of neurodegeneration. ${ }^{[50]}$

\section{Lipid Isomerization}

In the recent years geometrical isomerization of fatty acid residues has been recognized as a radical-based modification that can have a biological meaning.

In organic chemistry it is well known that several radical species catalyze the cis to trans isomerization by the formation of the most stable trans structure. The chemical transformation occurs via the additionelimination of thiyl radicals to the double bond, as shown in Scheme 2 for a monounsaturated substrate and a thiyl radical.

On the other hand, when a double bond is considered in biology, the cis geometry $(Z)$ is ubiquitously found and this structure is not considered as unstable from a biological point of view. Neither isomerization process and formation of stable trans lipid structures were thought to occur in eukaryotes. All structural and metabolic activities of mono- and polyunsaturated fatty acid (MUFA and PUFA) moieties are based on the common feature of cis geometry. The cis requisite is strictly controlled during lipid biosynthesis by desaturase enzymes, which in fact produce double bonds from saturated structures in a regiospecific and stereoselective manner.[51,52] 
Lipid cis/trans isomerization is a field of multidisciplinary research. In lipid chemistry related to nutrition, cis/trans isomeric mixtures are described as products during partial hydrogenation or deodorization of fats utilized in food industry. ${ }^{[53,54]}$ It must be demonstrated that in this case, geometrical isomers are formed together with positional trans isomers, the latter having the double bond positions shifted in the adjacent carbon atom, with respect to the natural cis isomer position. Investigations in nutrition and epidemiological studies pointed out some harmful effects of unnatural lipids for human health, ${ }^{[55]}$ and motivated a crusade to amend food labeling in USA by 2006, thus including trans fat information in the nutritional facts. [56] In microbiological research, it is known that trans lipids are formed in some bacteria by an enzymatic cis-trans isomerization during adaptation responses. [57] Trans fatty acids cannot be synthesized in eukaryotic cells. It is very important to note that in biochemistry trans fatty acid isomers are well known to produce perturbation effects on cell membrane arrangement and lipid enzymatic cascades.[58-62]

A few years ago, the general view of the lipid cis double bonds was that they are the natural structure, whereas trans isomers could occur in eukaryotic cells only after a dietary supplementation of chemically modified fats. More recently, the trans geometry in natural lipids was reconsidered, addressing a crucial question: can trans lipids be generated from two different pathways, namely, from foods, that we called the exogenous path, and from a radical isomerization, that we called the endogenous path, occurring during the metabolic functioning?

This question could arise from the known chemical reactivity of double bonds under radical conditions. In fact, thiyl radicals $\mathrm{RS}^{\bullet}$ are known to be some of the most effective species to catalyze the cis-trans isomerization process. ${ }^{63,64]}$ The connection with a biological process could be initially found by the consideration that thiyl radicals are generated from thiols under the radical stress, in particular because of the 'radical repair reaction', as well as during the activity of some enzymes (reaction 7).[65]

$\mathrm{R}^{\bullet}+\mathrm{RSH} \rightarrow \mathrm{RH}+\mathrm{RS}^{\bullet}$

Considering the possibility of lipid isomerization occurring in biological systems, the chemical biology approach was applied by combining the following aspects:

i) design of biomimetic models such as model membranes, to study trans lipid formation in the presence of biologically meaningful sulfur-containing compounds, examining different radical initiations and conditions; ii) mechanistic and product studies on lipid isomerization in homogenous and heterogeneous systems, to gather kinetic and thermodynamic information on the isomerization steps and product formation; this part can be associated with computational chemistry for getting predictive indications about mechanisms, and help to interpret data;

iii) synthetic studies to build up a trans lipid library to be used in lipidomics and biological research.

\subsection{Lipid Isomerization in Model Membranes}

Liposomes such as multilamellar vesicles (MLV) and small or large unilamellar vesicles (SUV, LUV), are widely accepted as models of membrane lipid assembly (see Fig. 1B). After an initial use of MLV vesicles, the choice was oriented to large unilamellar vesicles obtained by an extrusion technique (LUVET) for their close relationship with cell membranes, due to the presence of a single bilayer.[66,67] They can be prepared with quite a uniform diameter depending upon the polycarbonate filter size used for the extrusion. Vesicles of 100 $\mathrm{nm}$ diameter form an almost transparent suspension, which is also suitable for studies under photolytic conditions. ${ }^{68]}$

The aqueous and lipid phases are the two distinct compartments of this nonhomogeneous system. There are several features to be taken into account for examining the reactivity of this system towards free radicals:

i) the characteristic supramolecular arrangement of the lipid assembly, with the fatty acid chains of phospholipid molecules that form the hydrophobic core of the model membrane, and the polar heads that face the aqueous internal and external phases;

ii) the partition coefficient of compounds added in the system, which influences the distribution of the reactive species in the two compartments;

iii) in particular, the location of the initiation step, i.e. where the formation of an ini- tial radical species, able to abstract the $\mathrm{H}$-atom from the thiol group, occurs.

As far as the lipid organization is concerned, there is a precise arrangement of the hydrophobic core, which can influence the position of the double bonds in the layer and the reactivity of the different fatty acids to the radical attack. This was found to be the case in the double bond isomerization, studied with an amphiphilic thiol, 2-mercaptoethanol, as well as other diffusible thiols, such as $\mathrm{H}_{2} \mathrm{~S}$, [69] that are able to move without restriction from the aqueous phase to the lipid bilayer, and vice versa. A regioselective process resulted since the double bonds are not involved at the same extent by the radical isomerization. In particular, using vesicles made of egg yolk lecithin, it was possible to demonstrate that the double bonds located closest to the membrane polar region are the most reactive towards the attack of diffusing thiyl radicals. ${ }^{[70]}$ In the case of linoleic acid residues in vesicles, the double bond in position 9 was more reactive than that in position 12. Also arachidonic acid residues in vesicles were more reactive than oleic and linoleic acids, and two positions, i.e. the double bonds in 5 and 8, over the four present in this structure, were transformed preferentially. The work in the models highlighted arachidonic acid residues as very important markers in membrane phospholipids, in particular for distinguishing endogenous trans isomers, formed by radical processes, from the exogenous trans isomers, derived from dietary contribution. ${ }^{[66-71]}$

The partition coefficient of sulfurcontaining compounds used in the vesicle model and the compartment where the radical initiation step occurs, play a role in the isomerization outcome. Hydrophilic, lipophilic and amphiphilic compounds have a different behavior and the combination between the thiol and the radical initiator can be a crucial step. In biomimetic models, a variety of biologically relevant sulfurcontaining compounds, such as cysteine, glutathione, methionine, lipoic and dihydro lipoic acids, ${ }^{[72]}$ were examined under different radical initiating conditions (Fig. 4).
Fig. 4. Diffusion of thiyl radical species in the liposomes and cistrans isomerization of phospholipids

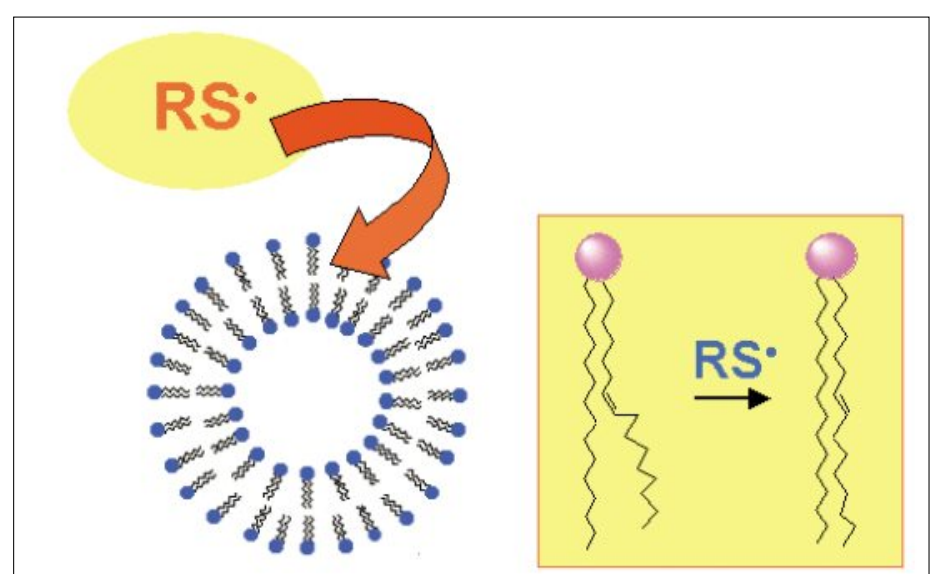


Radical initiation exclusively occurring in the aqueous compartment was obtained by two methodologies:

i) thermal decomposition of a hydrophilic azocompound, which can also have a biological significance, simulating the 'repair' hydrogen-donation reaction given by thiols, such as cysteine and glutathione, towards carbon-centered radicals;

ii) $\gamma$-irradiation of aqueous systems which gives $\mathrm{e}_{\mathrm{aq}}{ }^{-}, \mathrm{HO}^{\bullet}$, and $\mathrm{H}^{\bullet}$ as reactive species, and the possibility of selection of a specific species under appropriate conditions. ${ }^{[66,67]}$

By examining different combinations of thiols and initiators, it was established that hydrophilic compounds, such as cysteine, combined with a hydrophilic thermal initiator, did not give any isomerization of the lipid bilayer.[70] This behavior was easily explained by the fact that hydrophilic thiyl radicals are not able to enter the lipid bilayer, and therefore cannot reach the lipid double bonds.

On the other hand, under $\gamma$-radiolysis conditions lipid isomerization occurred. This radical damage was found to be caused by hydrogen atoms $\mathrm{H}^{\bullet}$ that are able to attack the sulfur moiety of the amino acid, and produce a desulfurization reaction. ${ }^{[73,74]}$ It is very interesting that this reactivity represents the molecular basis of a tandem lipid-protein damage, which is the subject of another paper of this CHIMIA issue (Chatgilialoglu and coworkers, p. 721-727).

Computational chemistry can offer a very important contribution to the mechanistic studies on fatty acid transformations by free radical processes. It allows thermochemical data, such as heats of formation, to be calculated with an accuracy of $\sim 1$ $\mathrm{kcal} / \mathrm{mol}$. Experimental heats of formation for radicals are difficult to obtain and can be in error. A disagreement between reliable theoretical and experimental data of more than $3-4 \mathrm{kcal} / \mathrm{mol}$ is usually a strong indication that experimental results need to be reviewed and perhaps re-interpreted. Unlike experiments, computations can locate and study transition-state structures.[75-77] The energies of the transition states relative to the reactants can be translated in relative kinetics predicting what process or processes among several competing ones maybe favored energetically. This was applied to cis-trans isomerization in comparison with experimental data ${ }^{[64]}$ and can be extended to other thiyl radical species generated from biologically relevant environment (as for example those derived from S-containing residues in peptides or proteins) as well as to polyunsaturated substrates, in order to evaluate competitivity from other processes, such as $\mathrm{H}$-abstraction or H-transfer, occurring intra- or intermolecularly.

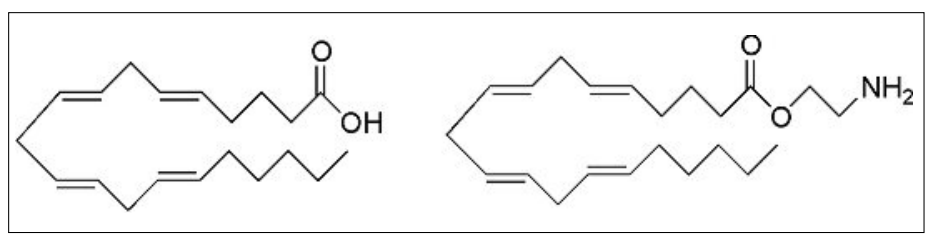

Fig 5. All-trans arachidonic acid and all-trans anandamide

\section{All trans Lipid Strategy}

The relevance of the double bond geometry for the biological activity of unsaturated fatty acids has been addressed by several studies on cell cultures and organisms. For example the common monounsaturated FA oleic acid in its free form specifically activates a number of enzymes such as oleic acid dependent PKC (protein kinase C), ${ }^{[78]}$ PLD (phospholipase D) and could act as an intracellular messenger causing $\mathrm{Ca}^{2+}$ mobilization in human platelets, ${ }^{, 79]}$ while the respective saturated fatty acid (stearic acid) and the trans-counterpart (elaidic) are inactive. This is also true for PUFA such as arachidonic acid, linoleic acid and their enzymatic (cyclooxygenase, COX, and lipoxygenase, LOX) products that are involved in a variety of biological responses. It should be pointed out that trans FA derived from PUFA are not an homogenous group and might have distinct biological activities. For instance, the 14-trans arachidonic acid isomer decreased platelet aggregation, ${ }^{[00]}$ whereas the 5-trans isomer did not.[81] The presence of trans fatty acids in living systems has been reported in a number of studies where the conditions are such that they arise endogenously by effect of radical stress. In these in vitro and in vivo cases, mono trans arachidonic acid isomers have been detected. $[67,71,82]$

The biological effects connected to the lipid geometry have suggested an antisense strategy focusing on the all-trans geometry, which is the opposite of cis configuration present in natural lipids; although it is highly improbable that these compounds could be formed endogenously by exhaustive isomerization, their study in comparison to their natural cis-counterparts can provide valuable information for the role of the double bond geometry by considering the interference in major physio-pathological biological actions. The synthesis of these compounds can be accomplished using the thiyl radical-catalyzed methodology, which was found to convert specifically the cis double bond into their geometrical trans counterparts without causing double bond shift. ${ }^{[83]}$ A number of trans fatty acids, such as all-trans arachidonic acid, all-trans linoleic acid as well as the respective derivatives of biologically important signaling molecules, namely all-trans arachidonoylethanolamide (all-trans anandamide) and all-trans linoleoylethanolamide, have been prepared so far (Fig. 5).
Anandamide is the most studied endocannabinoid which exerts its biological roles by binding to cannabinoid receptors (CB1, CB2) and it is then inactivated through degradation by the action of a fatty acid amidohydrolase (FAAH), inside the cell. Platelets were used as an experimental cell model to study the effects of trans fatty acids and derivatives in comparison to their cis counterparts. Platelets play an important role in maintaining vascular homeostasis and their function is modulated by cis unsaturated fatty acids, and their metabolic products, while trans FA have been accused of causing atherogenesis. [84]

Arachidonic acid induces platelet aggregation mainly through its conversion to tromboxane $\mathrm{A}_{2}\left(\mathrm{TXA}_{2}\right)$ which is then released and, by binding to its receptors, activates PLC (phospholipase C); subsequently, intracellular calcium is increased and amplifies platelet aggregation which in turn may cause further release of $\mathrm{TXA}_{2}$ and adenosine diphosphate (ADP) that leads to irreversible aggregation. It should be mentioned that platelet stimulation by thrombin results in $\mathrm{TXA}_{2}$ release while platelet activating factor (PAF) activates platelets through a TXA 2 independent pathway.

All-trans arachidonic acid was found to have a dual effect on platelets. At high concentrations $\left(>10^{-4} \mathrm{M}\right)$ aggregation was observed, while the respective cis acid had a similar effect at approximately ten times lower concentration. It is noteworthy that at lower concentrations all trans arachidonic acid inhibited the aggregation induced by the strong platelet agonist PAF $\left(4 \times 10^{-10}\right.$ $\mathrm{M})$ with an $\mathrm{IC}_{50} \sim 6 \mu \mathrm{M}$, but not that induced by thrombin. Arachidonic acid and all-trans arachidonic acid methylesters, as well as the respective saturated isomer (arachidic acid) had no effect. These results suggest that the presence of double bond and the free form of the FA are necessary for the activity. Similar results were obtained with all-trans linoleic acid.

On the other hand, anandamide caused platelet aggregation at a concentration-dependent manner through its conversion to AA, apparently by the action of FAAH. ${ }^{[85]}$ Indeed aggregation was inhibited by PMSF (phenylmethanesulphonyl fluoride, a serine protease inhibitor) and the rather specific FAAH inhibitor, URB597. Interestingly all-trans anandamide behaved like $t$-AA causing aggregation at high concentrations and inhibiting platelet aggregation induced 
by PAF at lower concentrations $\left(\mathrm{IC}_{50} \sim 4.7\right.$ $\mu \mathrm{M}) .{ }^{\left[{ }^{[8]}\right]}$ The aggregation was not inhibited by FAAH inhibitors, suggesting that a different mechanism was involved.

Nevertheless, all-trans anandamide as well as all-trans linoleoylethanolamide were good substrates for FAAH when platelet homogenate or Tetrahymena thermophila cell homogenate was used as enzymatic source.[86]

The antisense strategy carried with alltrans lipids indicated that the effects obtained by such structural change could interfere with the pathways followed by their (normal) cis counterparts involved in very important functions. The aim of these studies is to contribute to the understanding of the role and the importance of the double bond configuration in biological systems. In addition, the possibility that all-trans AA and all-trans anandamide might be proved rather specific PAF inhibitors is very interesting and needs further investigation since PAF is a very potent mediator of inflammation.

\section{Conclusions}

The combination of studies in lipidomics with those of free radical modifications concerning lipids is promising for the understanding of the molecular basis in biology and medicine, either involved in ageing and in several pathologies or in the physiological metabolic functioning of membranes and signaling pathways. The chemical biology approach is important not only for fostering collaborations among different fields, but also for improving a common language, in order to share information from life science disciplines. This will allow chemical properties and mechanisms to become a useful basis for understanding biological processes, contributing to the solution of puzzling questions on free radical stress and suggesting strategies for medical applications. In this respect, as demonstrated by peroxidation and more recently, by isomerization processes, the field of free radicals and their applications to biology and medicine is not only increasing the scientific role of chemistry in life sciences, but also hopefully contributing to a better social perspective of chemistry for its role in the improvement of health and quality of life.

\section{Acknowledgement}

The support and sponsorship by COST Action CM0603 on 'Free Radicals in Chemical Biology (CHEMBIORADICAL)' are kindly acknowledged.

Received: July 11, 2008

[1] M. R. Wenk, Nat Rev Drug Discov. 2005, 4, 594.
[2] G. Van Meer, $E M B O J . \mathbf{2 0 0 5}, 24,3159$.

[3] E. Niki, Y. Yoshida, Y. Saito, N. Noguchi, Biochem. Biophys. Res. Commun. 2005, 338, 668.

[4] B. Halliwell, J. M. C. Gutteridge, 'Free Radicals in Biology and Medicine', 3rd ed., Oxford University Press Inc., New York, 1999.

[5] C. Ferreri, C. Chatgilialoglu, ChemBioChem 2005, 6, 1722 .

[6] C. Chatgilialoglu, C. Ferreri, Acc. Chem Res. 2005, 36, 441.

[7] N. A. Porter, B. A. Weber, H. Weenen, J. A. Khan, J. Am. Chem. Soc. 1980, 102, 5597.

[8] N. A. Porter, R. A. Wolf, E. M. Yarbro, H. Weenen, Biochem. Biophys. Res. Commun. 1979, 89, 1058.

[9] L. R. C. Barclay, Can. J. Chem. 1993, 71, 1.

[10] K. A Tallman, B. Roschek, Jr., N. A. Porter, J. Am. Chem. Soc. 2004, 126, 9240.

[11] B. Katušin-Ražem, D. Ražem, J. Phys. Chem. A 2000, 104, 1482.

[12] H. Esterbauer, G. Striegl, H. Puhl, M Rotheneder, Free Radic. Res. Commun. 1989, 6, 67.

[13] H. C. Yeo, J. Liu, H. J. Helbock, B. N. Ames, Methods Enzymol. 1999, 300, 70.

[14] B. Mihaljević, B. Katušin-Ražem, D. Ražem, Free Radical Biol. Med. 1996, 21, 53.

[15] O. Raveh, I. Pinchuk, E. Schnitzer, M. Fainaru, Z. Schaffer, D. Lichtenberg, Free Radical Biol. Med. 2000, 29, 131.

[16] S. J. Stohs, D. Baggi, Free Radical Biol. Med. 1995, 18, 321.

[17] G. Van Ginkel, A. Sevanian, Methods Enzymol. 1994, 233, 273.

[18] T.Lundström, H. Christensen, K. Sehested, Rad. Phys. Chem. 2004, 69, 211.

[19] Z. Cheng, Y. Li, Chem. Rev. 2007, 107, 748.

[20] G. Minotti, S. D. Aust, J. Biol. Chem 1987, 2621098.

[21] B. Mihaljević, D. Ražem, Rad. Phys. Chem. 2003, 67, 269.

[22] J. D. Rush, W. H. Koppenol, FEBS Lett. 1990, 275, 114

[23] N. A. Porter, S. E. Caldwell, K. A. Mills, Lipids 1995, 30, 277.

[24] G. Spiteller, Mol. Biotechnol. 2007, 37, 5.

[25] T. L. Kaduce, P. H. Figard, R. Leifur, A. A Spector, J. Biol. Chem. 1989, 264, 6823.

[26] P. Blanc, A. Revol, H. Pacheco, Nutr. Res 1992, 12, 833 .

[27] C. Brandsch, K. Eder, Br. J. Nutr. 2004 92, 267.

[28] C. Schneieder, D. A. Pratt, N. A. Porter, A. R. Brash, Chem. Biol. 2007, 14, 473.

[29] I. G. Denisov, T. M. Makris, S. G. Sligar, I. Schlichting, Chem. Rev. 2005, 105, 2253

[30] J. L. Witztum, D. Steinberg, J. Clin. Invest 1991, 88, 1785

[31] J. A. Berliner, M. Navab, A. M. Fogelman, J. S. Frank, L. L. Demer, P. A. Edwards, A. D. Watson, A. J. Lusis, Circulation 1995, 91, 2488.

[32] G. L. Milne, N. A. Porter, Lipids 2001, 36 , 1265

[33] H.-P. Deigner, A. Hermetter. Curr. Opin. Lipidol. 2008, 19, 289.

[34] T. Yamase, J. Mater. Chem. 2005, 15, 4773.
[35] C. Boglio, K. Micoine, E. Derat, R. Thouvenot, B. Hasenknopf, S. Thorimbert, E. Lacôte, M. Malacria, J. Am. Chem. Soc. 2008, 130, 4553.

[36] K. Micoine, B. Hasenknopf, S. Thorimbert, E. Lacôte, M. Malacria, Org. Lett. 2007, 9, 3981.

[37] S. Bareyt, S. Piligkos, B. Hasenknopf, R. Gouzerh, S. Thorimbert, E. Lacôte, M Malacria, J. Am. Chem. Soc. 2005, 127 , 6788.

[38] G. Spiteller, Ann. N. Y. Acad. Sci. 2005 , 1043, 355.

[39] G. Spiteller, Free Radical Biol. Med. 2006, 41, 362.

[40] G. Paradies, G. Petrosillo, M. Pistolese, N. Di Venosa, D. Serena, F. M. Ruggiero, Free Radical Biol. Med. 1999, 27, 42.

[41] R. H. Houtkooper, F. M. Vaz, Cell. Mol. Life Sci. 2008, 65, 1420.

[42] Y. Y. Tyurina, V. A. Tyurin, M. W. Epperly, J. S. Greenberger, V. E. Kagan, Free Radical Biol. Med. 2008, 44, 299.

[43] G. O. Fruhwirth, A. Loidl, A. Hermetter, Biochim. Biophys. Acta 2007, 1772, 718.

[44] W. A. Pryor, J. P. Stanley, J. Org. Chem. 1975, 40, 3615.

[45] H. Esterbauer, R. G. Schaur, H. Zollner, Free Radical Biol. Med. 1991, 11, 81 .

[46] B. S. Kristal, B. K. Park, B. P. Yu, J. Biol. Chem. 1996, 271, 6033

[47] J. N. Keller, R. J. Mark, A.J. Bruce, E. Blanc, J. D. Rothstein, K. Uchida, G Waeg, M. P. Mattson, Neuroscience, 1997, 80, 685 .

[48] J. W. Crabb, J. O’Neil, M. Miyagi, K. West, H. F. Hoff, Protein Sci. 2002, 11, 831

[49] S. Poggioli. J. Mary, H. Bakala, B. Friguet, Ann. N. Y. Acad. Sci. 2004, 1019, 211.

[50] I. Kruman, A. J. Bruce-Keller, D. Bredesen, G. Waeg, M. P. Mattson, J. Neurosci. 1997, 17, 5089 .

[51] B. G. Fox, K. S. Lyle, C. E. Rogge Acc. Chem. Res. 2004, 37, 421.

[52] 'Cis-trans Isomerization in Biochemistry' Ed. C. Dugave, Wiley-VCH, Weinheim, 2006.

[53] Y. Kitayama, M. Muraoka, M. Takahashi, T. Kodama, E. Takahashi, M. Okamura, $J$. Am. Oil Chem. Soc. 1997, 74, 525.

[54] 'Trans Fatty Acids in Human Nutrition', Eds. J. L. Sébédio, W. W. Christie, The Oily Press, Dundee, 1998.

[55] D. Mozaffarian, M. B. Katan, A. Ascherio, M. J. Stampfer, W. C. Willett, N. Engl. J. Med. 2006, 354, 1601.

[56] S. Okie, N. Engl. J. Med. 2007, 356 2017.

[57] H. Keweloh, H. J. Heipieper, Lipids 1996 , $31,129$.

[58] G. M. Helmkamp, Biochemistry 1980, 19, 2050.

[59] D. J. Siminovitch, P. T. T. Wong, H. H Mantsch, Biochemistry 1987, 26, 3277.

[60] O. Berdeaux, J. M. Chardigny, J. L. Sébédio, J. Lipid Res. 1996, 37, 2244.

[61] F. A. Kummerow, Q. Zhou, M. M. Mahfouz, Am. J. Clin. Nutr. 1999, 70 , 832.

[62] A. M. Giudetti, A. C. Beynen, A. G. Lemmens, Brit. J. Nutr. 2003, 90, 887.

[63] C. Chatgilialoglu, A. Altieri, H. Fischer, $J$. Am. Chem. Soc. 2002, 124, 12816. 
[64] C. Chatgilialoglu, A. Samadi, M. Guerra, H. Fischer, ChemPhysChem 2005, 6, 286.

[65] C. Ferreri, S. Kratzsch, L. Landi, O. Brede, Cell. Mol. Life Sci. 2005, 62, 834.

[66] C. Ferreri, F. Sassatelli, A. Samadi, L. Landi, C. Chatgilialoglu, J. Am. Chem. Soc. 2004, 126, 1063.

[67] C. Ferreri, M. R. Faraone-Mennella, C. Formisano, L. Landi, C. Chatgilialoglu, Free Radical Biol. Med. 2002, 33, 1516.

[68] C. Ferreri, S. Pierotti, A. Barbieri, L. Zambonin, L. Landi, S. Rasi, P. L. Luisi, F. Barigelletti, C. Chatgilialoglu, Photochem. Photobiol. 2006, 82, 274.

[69] I. N. Lykakis, C. Ferreri, C. Chatgilialoglu, Angew. Chem. Int. Ed. 2007, 46, 1914.

[70] C. Ferreri, C. Costantino, L. Perrotta, L. Landi, Q. G. Mulazzani, C. Chatgilialoglu, J. Am. Chem. Soc. 2001, 123, 4459.

[71] C. Ferreri, F. Angelini, C. Chatgilialoglu, S. Dellonte, V. Moschese, P. Rossi, L. Chini, Lipids 2005, 40, 661.

[72] C. Chatgilialoglu, L. Zambonin, A. Altieri, C. Ferreri, Q. G. Mulazzani, L. Landi, Free Radical Biol. Med. 2002, 33, 1681.

[73] C. Ferreri, C. Chatgilialoglu, A. Torreggiani, A. M. Salzano, G. Renzone, A. Scaloni, J. Prot. Res. 2008, 7, 2007.

[74] O. Mozziconacci, K. Bobrowski, C. Ferreri, C. Chatgilialoglu, Chem. Eur. J. 2007, 13, 2029.

[75] A. Nicolaides, T. Matsushita, H. Tomioka, J. Org. Chem. 1999, 64, 3299.
[76] A. Nicolaides, L. Radom, J. Am. Chem. Soc. 1997, 119, 11933.

[77] A. Nicolaides, W. T. Borden, J. Am. Chem. Soc. 1992, 114, 8682.

[78] W. A. Khan, G. Blobe, A. Halpern, W. Taylor, W. C. Wetsel, D. Burns, C. Loomis, Y. A. Hannun, J. Biol. Chem. 1993, 268, 5063.

[79] A. Siafaka-Kapadai, D. J. Hanahan, M. A. Javors, J. Lipid Mediat. Cell Signal. 1997, $15,215$.

[80] G. J. N. Egmond, H. J. J. Pabon, D. A vanDorp, Rec. Trav. Chim. Pays-Bas. 1977, 96, 172.

[81] O. Berdeaux, J.-M. Vatèle, T. Eynard, M. Nour, D. Poullain, J. P. Nöel, J. M Chardigny, J. L. Sébédio, Chem. Phys. Lipids 1995, 75, 71.

[82] C. M. Zghibeh, G. Raj, C. D. Poff, J. R. Falck, M. Balazy, Anal. Biochem. 2004, 332, 137.

[83] D. Anagnostopoulos, C. Chatgilialoglu, C. Ferreri, A. Samadi, A. Siafaka-Kapadai, Bioorg. Med. Chem. Lett. 2005, 15, 2766.

[84] M.A. Thijssen, R.P. Mensink, Handb. Exp. Pharmacol. 2005, 165.

[85] S. Braud, C. Bon, L. Touqui, C. Mounier, FEBS Lett. 2000, 471, 12.

[86] C. Ferreri, D. Anagnostopoulos, I. N. Lykakis, C. Chatgilialoglu, A. SiafakaKapadai, Bioorg. Med. Chem. in press. 